\title{
PRIMARY NASAL TUBERCULOSIS - A CASE REPORT
}

\author{
Mohan Kameswaran,* R.S Anand Kumar,* Sathiya Murali,* S. Raghunandan,* P. Vijaya Krishnan**
}

\begin{abstract}
During the past two decades, Tuberculosis - both pulmonary and extrapulmonary have re-emerged as a major health problem worldwide. Nasal tuberculosis may be primary, or secondary to pulmonary tuberculosis or facial lupus. However all of them are rare entities. Nasal tuberculosis should be considered in the differential diagnosis of chronic nasal granulomas. We report a case of primary nasal tuberculosis in an adult female who presented with a polypoidal lesion in the nasal cavity. The diagnosis was based upon smear study, histopathology, culture \& polymerase chain reaction. The patient successfully responded to antituberculous therapy and is presently disease free. Given the resurgence of tuberculosis in recent times, it is important that otolaryngologists remain aware of this rare clinical entity.
\end{abstract}

Keywords: Primary Nasal Tuberculosis, Polymerase Chain Reaction \& Anti-Tuberculous Therapy

\section{INTRODUCTION}

Due to modern chemotherapy, the incidence of upper respiratory tract tuberculosis had been steadily declining in the industrialized world for many decades. However since 1985, mycobacterial disease has been on the rise, especially among persons living in unhygienic surroundings and with immuno-compromised status, causing a resurgence of interest in finding a cure for this disease ${ }^{[1-3]}$. Nasal tuberculosis has always been considered secondary to tuberculosis of the lungs and in rare instances constituting a primary infection, usually due to inhalation of mycobacterium. A review of modern literature, suggests that more often than not, the disease seems to be primary ${ }^{[4,5]}$. It is more common in females than males, especially of middle age and in general seems to be rare (no more than 40 cases have been reported in recent world literature) ${ }^{[5-7]}$.

The first case of Primary Tuberculosis of the upper respiratory tract and nasal passages was presented to the Pathological Society of London by Clarke in $1852 .{ }^{(3)}$ Later in the 18th century, reviews published by Herzog described 20 cases of primary nasal

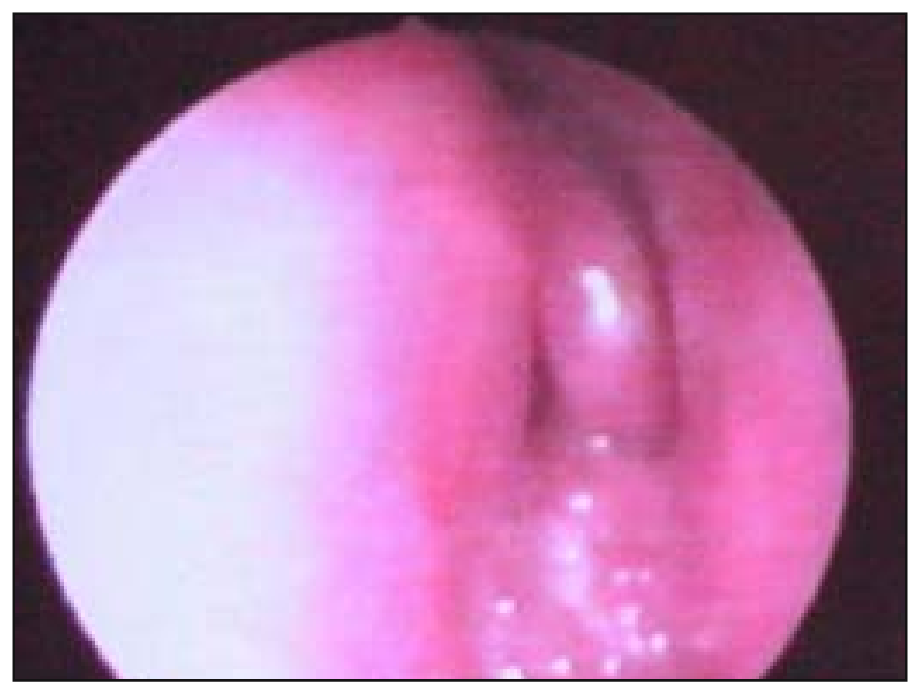

Fig I:Endoscopic view showing granular mass in the left nasal cavity tuberculosis among 80 cases of nasal tuberculosis ${ }^{[7]}$. In a review of the 20th-century medical literature published in 1997, Butt found only 35 cases of primary nasal tuberculosis ${ }^{[18]}$.

\section{CASE REPORT}

A 30 year old lady was referred by her general practitioner to our institution, for evaluation of a 8-week history of left nasal obstruction with occasional epistaxis. Her general practitioner had prescribed a course of broad spectrum antibiotics and antihistamine therapy for nonspecific rhinitis, but it did not relieve her symptoms.

The patient's general condition and systemic examination was normal. Anterior rhinoscopic examination revealed the presence of a sessile reddish polypoidal lesion arising from the anterior edge of the inferior turbinate in the left nasal cavity (Figure1). On probing, the lesion was not bleeding or friable, non compressible and non pulsatile. The adjacent mucosa over the turbinate and the mucosa over the septum were thick and hyperemic. Other ENT examination was normal.

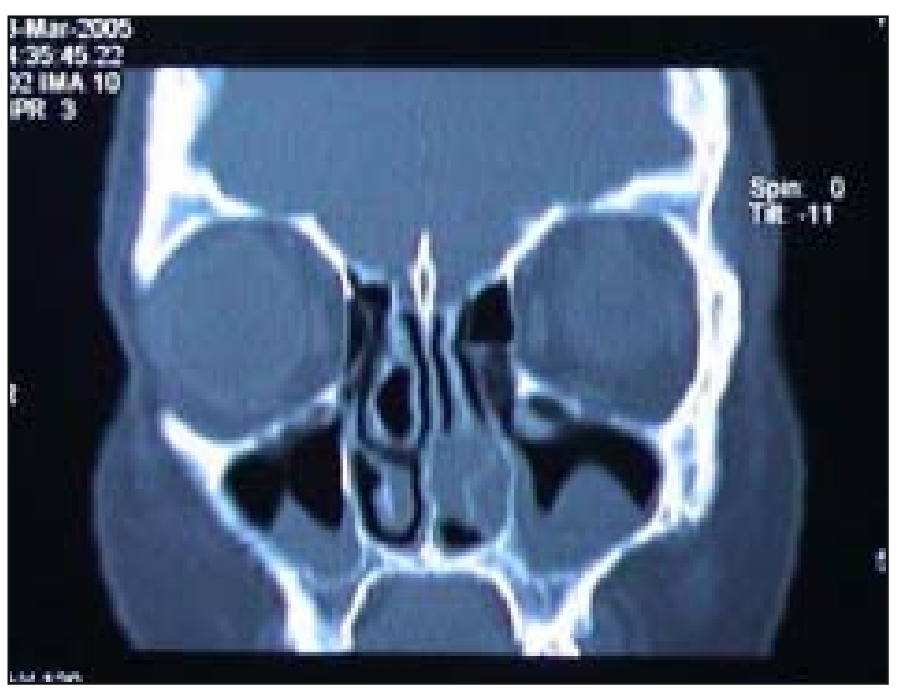

Fig II:CT scan paranasal sinuses coranal view showing mass in the left nasal cavity 
Hematological tests revealed her erythrocyte sedimentation rate was $20 \mathrm{~mm} / \mathrm{hr}$ by Westergren's method (normal: $<15 \mathrm{~mm} / \mathrm{hr}$ ). Her HIV status was negative and results of her renal and liver function tests were normal. Her three consecutive sputum smears were negative for acid-fast bacilli, as were subsequent cultures. Findings on computed tomography of the paranasal sinuses showed irregular hypertrophy of the inferior turbinate on the left side and mucosal thickening in the left maxillary sinus (Figure 2, 3). Findings of chest $\mathrm{x}$-ray were normal.

The granulomatous lesion was endoscopically biopsied under local anaesthesia. Minimal bleeding from the lesion was noted during the procedure. A provisional diagnosis of Granulomatous disease of the nose was considered. The diagnosis was proved to be Nasal Tuberculosis by smear study, histo-pathology, culture study and polymerase chain reaction for Mycobacterium tuberculosis.

Biopsy analysis revealed a caseating granulomatous lesion made up of epithelioid cells, lymphocytes, and a few giant cells (Figure 4). The stroma contained many lymphocytes and a few mucosal glands. Special staining for fungus, atypical mycobacterium and lepra bacilli were negative. Typical acid-fast bacilli were noted on Ziehl-Neelsen staining. Culture for Mycobacterium in Lowenstein-Jensen medium was positive after 4 weeks . A positive Polymerase Chain Reaction for mycobacterium tuberculosis clinched the diagnosis of Nasal Tuberculosis. Since no other focus of the disease was identified, a final diagnosis of primary nasal tuberculosis was arrived at.

The patient was administered Anti Tuberculous Therapy (multidrug regimen) with isoniazid, rifampin, pyrazinamide and ethambutol for 2 months, followed by isoniazid and rifampin for 4 months. The patient underwent regular follow-up, including comprehensive systemic and otolaryngological examination with nasal endoscopy, and presently she remains disease-free at 9 months of follow up.

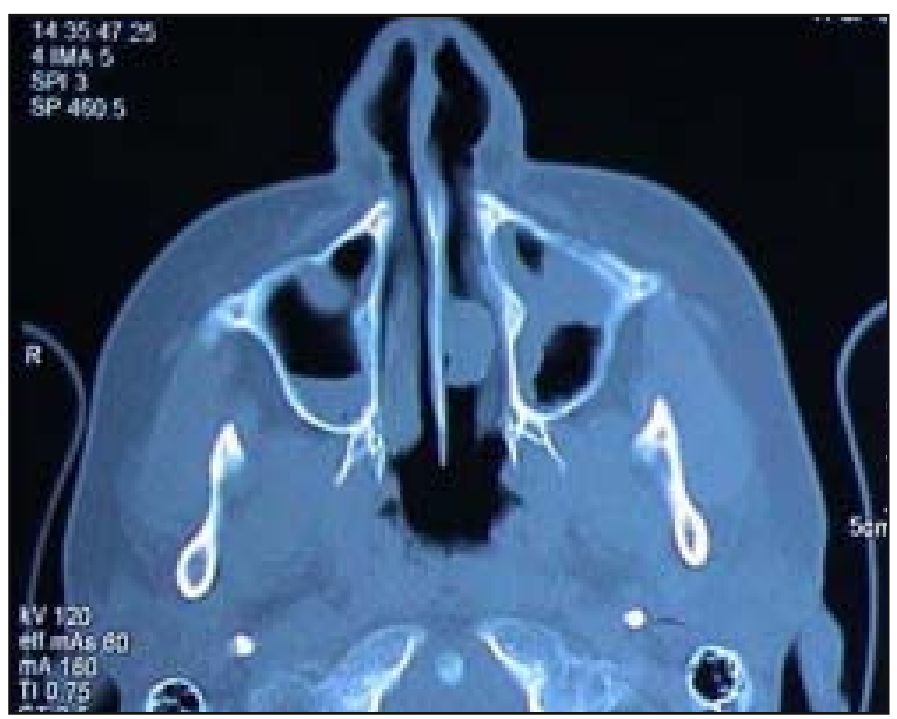

Fig III:CT scan paranasal sinuses axial view showing mass in the left nasal cavity

\section{DISCUSSION}

The striking increase in tuberculosis during recent years is characterized by some new features, such as emerging resistance to formerly effective antituberculosis drugs, the aggressive nature of the disease in patients infected with the human immunodeficiency virus (HIV) and the increased incidence of the extrapulmonary disease in the 25 to 44 year-old group, which accounts for two thirds of the recorded cases ${ }^{[1,8]}$. In spite of this increase, nasal tuberculosis remains a rare entity, presenting diagnostic and therapeutic problems to the physician ${ }^{[5]}$. M. tuberculosis may be introduced into the nasal cavity either by local infiltration due to finger contact or by the inhalation of infected droplets or dust. The clinical picture is characterized by unilateral or bilateral nasal obstruction, accompanied by mucoid or mucopurulent rhinorrhea and postnasal discharge.

Other symptoms might include epistaxis, itching and sneezing ${ }^{[9]}$. By anterior rhinoscopy, nasal tuberculosis appears as a discrete, soft granular swelling of the nasal septum, which often ulcerates ${ }^{[10]}$. Without treatment, progress is rapid, leading to ulceration of the lesion and finally perforation of the nasal septum. Involvement of the bony septum is rare. However, other sites are occasionally involved, such as the turbinates and the floor of the nose. Extension to the maxillary sinus although reported seems to be rare $^{[11]}$. Differential diagnosis includes granulomatous disease of the nose such as leprosy, mycoses, sarcoidosis ${ }^{[12]}$ malignancies ${ }^{[6]}$ and skin disease (lupus erythematosus) ${ }^{[13]}$. Wegener's granulomatosis and midline malignant reticuloma, although rarely encountered, should always be considered as possible causes of nasal granuloma. The diagnosis is established by the presence of caseating granulations, Langhans' type giant cells and acid/ alcohol-fast bacilli on histolopathological examination ${ }^{[14]}$. The gold standard for diagnosis is a positive culture of M.tuberculosis from tissue sample. To confirm the diagnosis, the M. tuberculosis must be identified in the tissue's specimens obtained during excisional biopsy or from tissue cultures submitted at the same

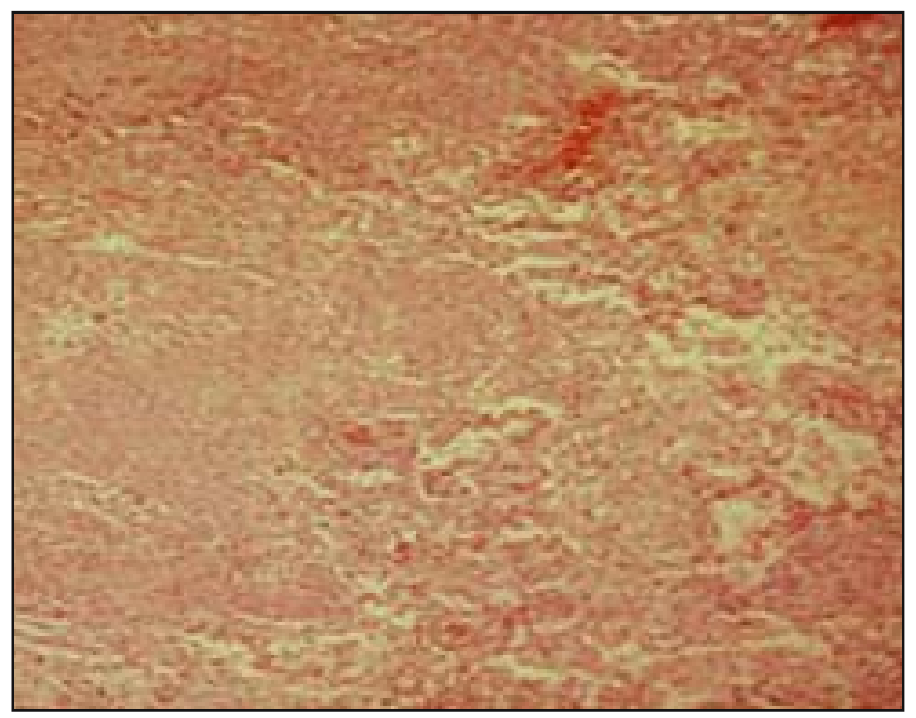

Fig IV:Histopathological picture showing caseating granulomatous lesion Epithelioid cells, Lymphocytes with few langhans gaint cells 
time, though in many cases this is quite difficult to achieve. The need for a more rapid and accurate diagnosis has led to molecular diagnostic methods. Kapur et al. ${ }^{[15]}$ have used a polymerase chain reaction, DNA-sequence-based strategy that could permit identification of mycobacterial species, as well as mutations associated with antimicrobial resistance in less than $48 \mathrm{hrs}$.

As nasal tuberculosis may be secondary, a primary site of the disease, especially in the lungs, must always be sought for by both clinical and radiological investigations. Though primary nasal tuberculosis is not particularly infectious, investigation of contacts is always recommended. Surgical treatment is not necessary in localized nasal disease, and the standard antituberculosis therapy is usually adequate to obtain complete cure of the disease. It should be mentioned, however, that drug resistance must always be considered during treatment [7]. Surgical intervention is occasionally needed to treat local complications, such as extensive nasal scarring. Adequate local treatment, including nasal douching and removing of crusts, is also important. The basic principles that underlie the treatment of pulmonary tuberculosis also apply to extrapulmonary forms of the disease, and increasing clinical experience seems to indicate that 6- to 9-month short course regimens are very effective ${ }^{[16]}$. We administered a 6-month course of therapy that resulted in disappearance of the granulation tissue from the nose.

In conclusion, we believe that although primary nasal tuberculosis remains a rare clinical entity, the Otolaryngologist should always consider it in the differential diagnosis of patients with granulomatous lesions of the nose. The clinician will have to be aware of the new aspects of the disease, as regards to the recent epidemiological features, current diagnosis and management.

\section{REFERENCES}

1. Cantwell MF, Snider DE, Cauthen GM, Onorato IM: Epidemiology of tuberculosis in the United States, 1985 through 1992. JAMA 1994;272:535-539.

2. Williams RG, Douglas-Jones T: Mycobacterium marches back. J Laryngol Otol 1995;109:5-13.

3. Al-Serhani AM: Mycobacterial infection of the head and neck: Presentation and diagnosis. Laryngoscope 2001;111:2012-2016.0

4. Cleary KB, Batsakis JG: Mycobacterial disease of the head and neck: Current perspective. Ann Otol Rhinol Laryngol 1995; 104:830833.
5. Butt AA: Nasal tuberculosis in the 20th century. Am J Med Sci 1997;313:332-335.

6. Sim DW, Crowther JA: Primary nasal tuberculosis masquerading as a malignant tumour. J Laryngol Otol 1988;102:1150-1152.

7. Johnson IJ, Soames JV, Marshall HF: Nasal tuberculosis - An increasing problem? J Laryngol Otol 1995;109:326-327.

8. Springett V, Darbyshire J, Nunn A, Sutherland I: Changes in tuberculosis notification rates in the white ethnic group in England and Wales 1953-1982. J Epidemiol Commun Health 1988; 42:370376.

9. Blanco Aparicio M, Verea-Hernando H, Pombo F: Tuberculosis of the nasal fossa manifested by a polypoid mass. J Otolaryngol 1995;24:317-318.

10. Choi YC, Park YS, Jeon EJ, Song SH: The disappeared disease: Tuberculosis of the nasal septum. Rhinology 2000;38:90-102.

11. Jha D, Deka RC, Sharma MC: Tuberculosis of the maxillary sinus manifesting as a facial abscess. Ear Nose Throat J 2002;81:102104.

12. Hughes $R G$, Drake-Lee A: Nasal manifestations of granulomatous disease. Hosp Med 2001;62:417-421.

13. Duzgun N, Duman M, Sonel B, Peksari Y, Erdem C, Tokgoz, G: Lupus vulgaris in a patient with systemic lupus erythematosus and persistent IgG deficiency. Rheumatol Int 1997; 16:213-216.

14. Hup AK, Haitjema T, de Kuijper G: Primary nasal tuberculosis. Rhinology 2001;39:47-48.

15. Kapur V, Li L-L, Hamrick MR, Plikaytis BB, Shinnick TM, Telenti A, Jacobs WR, Banerjee A, Cole S, Yuen KY, Clarridge JE III, Kreiswirth BN, Musser JM: Rapid Mycobacterium species assignment and unambiguous identification of mutations associated with antimicrobial resistance in Mycobacterium tuberculosis by automated DNA sequencing. Arch Pathol Lab Med 1995;119:131138.

16. American Thoracic Society, Centers for Disease Control: Treatment of tuberculosis and tuberculosis infection in adults and children. Am J Respir Crit Care Med 1994;149:1359- 1374.

17. Waldman SR, Levine HL, Sebek BA, et al. Nasal tuberculosis: A forgotten entity. Laryngoscope 1981;91:11-16.

18. Butt AA. Nasal tuberculosis in the 20th century. Am J Med Sci 1997;313:332-5.

Address for correspondence

Madras ENT Research Foundation No. $1,1^{\text {st }}$ Cross street, Off $2^{\text {nd }}$ Main Road, Raja Annamalaipuram, Chennai - 600028 E-mail:merfmk@vsnl.net 